\title{
Improved ALPR system based on Smart License Plate Character Detection Algorithm
}

\author{
Rohollah Mazrae Khoshki ${ }^{1}$, Subramanian Ganesan ${ }^{2}$ \\ Electrical and Computer Engineering, Oakland University, Rochester, USA ${ }^{1,2}$
}

\begin{abstract}
This paper presents improved Automated License Plate Recognition (ALPR) system based on Smart License Plate Character Detection algorithm, this system is capable of distinguishing license plates under various conditions, such as distance from the camera, rotation angle between camera and vehicle and poor illumination condition (different weather condition, different lighting condition and physical tilted or damage of license plate). In our method, improved ALPR system has three main steps: 1) Image Enhancement, 2) Character Segmentation (Smart License Plate Character Detection), 3) Character Recognition. While in regular ALPR system there are four main steps: 1) Image Enhancement, 2) License Plate Location Extraction 3) Character Segmentation, 4) Character Recognition. In image enhancement introduce Multi-Scale Adaptive NICK thresholding method [1] to achieve all objects as character candidates in the binary image; otherwise we might lose license plate characters in an input image due to complex backgrounds. Single Pass Connected Component Labelling (CCL), put the label on detected objects then new design algorithm called "Smart License Plate Character Detection" finds the license plate characters regardless of location, shape and background colour of license plate. So we eliminate the license plate location extraction as complex and time consuming individual stage [16]. All the steps simulated in Matlab 2014A software and results show the accuracy and reliability of this proposed method.
\end{abstract}

Keywords: ALPR system, Connected Components Labelling (CCL), Character Segmentation, Character Recognition, Multi-Scale Adaptive NICK thresholding Method, Smart License Plate Character Detection.

\section{INTRODUCTION}

Intelligent Transportation System (ITS) helps us by managing the cameras on the road to detecting the vehicles based on their license plate. Automated License Plate Recognition (ALPR) system finds the vehicle license plate from images taken by camera [2, 16]. Many researchers have been done for ALPR system but improving of ALPR systems based on accuracy and computation time is still on demand. ALPR system should have high degree of reliability in different illumination situation such as low or high illumination during the day or different weather condition and angle between the cameras and the license plates. Previous ALPR system [2] has four main processing stages for recognition of license plate in input images according:

1) Image Enhancement

2) License Plate Location Extraction

3) Character Segmentation

4) Character Recognition

In this research we present new Improved ALPR system that has three main processing stages:

1) Image Enhancement

2) Character Segmentation (Smart License Plate Character Detection Algorithm)

3) Character Recognition

In [1] we introduced Multi-Scale Adaptive NICK method for thresholding and Binarization then Single Pass Connected Components Labelling (CCL) method labels all the license plate character candidates on the binary image. We design new algorithm "Smart License Plate Character Detection" that detects and segments license plate characters regardless of license plate location, shape and background colour that will be explain in detail. Main contributions on this research are design of new algorithm called: "Smart License Plate Character Detection".

The rest of this paper is structured as follows. Section 2 presents new algorithm that design to find license plate characters on the binary image regardless of license plate location, shape and background colour that we called: "Smart License Plate Character Detection" algorithm and shows simulation results and comparing the achieved results with other methods. Finally, Section 3 explains conclusion and summary.

\section{SMART LICENSE PLATE CHARACTER DETECTION ALGORITHM}

In USA, license plate has English Capital letter and Number characters without any dot and point then Connected Components Labelling (CCL) [10] is applicable for license plate characters detection. The algorithm detects the regions of connected pixels in an image and creating a symbolic matrix that identifies each region with an individual label as character candidates. Single Pass Connected Components Labelling [11] Algorithm applies to label binary image objects. One of the advantages of Single Pass Connected Components Labelling method is whenever you face by blank column in image you could extract connected component objects in real time till that point without need to scan whole the image. By implementation of this method in a real time system we could reach number of Objects, labelling, Area of each objects and number of merges in binary image. 
Character Segmentation is an important and vital step for extracting characters to feed the Optical Character Recognition (OCR) process. For most of character segmentation in ALPR, first license plate location must be extracted in the image and then character segmentation applied at license plate location as the region of interest, different techniques are developed for license plate location extraction [13-14].

In our proposed method "Smart License Plate Characters Detection" algorithm, license plate characters determined and classified regardless of license plate location, shape and background colour. License plate location extraction could be a complex and time consuming process step [1, 13-14] that will be eliminated in our proposed method as individual step. Smart License Plate Characters Detection, algorithm has three main steps:

- Objects Size and Aspect Ratio Filtering

- Initialization and Objects Distance Determination

- Consecutive Objects and Character Counting

At each step we eliminate and filtering false objects, at the end we reach license plate characters for each license plate separately in an image up to 2 license plates at input image. In this method license plate characters will be determined regardless of license plate location, shape and background colour that make this method more reliable and universal.

\section{A. Objects Size and Aspect Ratio Filtering}

License plate characters are capital English letters and numbers in standard size so Aspect Ratio value (Length versus Width value of character) is constant. We check size and aspect ratio of objects, Michigan license plate character has aspect ratio of 2.8 according to standard format. The aspect ratio could be different in each country/state based on different standard font type that use for license plate characters, so this aspect ratio initialize and adjust once in algorithm based on standard font type. Based on experimental results, specific range is determined for aspect ratio of license plate characters, because in degraded image aspect ratio might slightly change. Aspect ratio range for characters is calculated between [2.5 4] to cover all type of the input image including high quality and degraded images. Also number of foreground pixels per total number of pixels in character candidate objects is calculated that should be in the predefined broad range of [0.2 0.85] for standard characters to eliminate false character candidates. While measuring aspect ratio of all objects to check if they are in the predefined aspect ratio range, Length and Width of object also will be measured for further processing. Approximation distance between the camera locations versus the car location is determined then the size of license plate and characters in the image could be assumed in the broad range. Minimum size of characters in the $1333 \times 1000$ pixels assume 30 pixels, according to practical test minimum character size to be visible and detectable in image is 30 pixels to cover far distance car in input images. Maximum size of license plate characters roughly estimated as 2500 pixels when car occupy whole the image. In each step character's size assumption fully oversized to have different size of license plate characters, it must mention that for this algorithm there is no limitation for maximum size of character candidates. Approximation of character size helps the algorithm to work faster by elimination of out of size false objects, but even if the maximum size of objects increased the algorithm still will be worked properly without any problem and limitation. Finally objects will be filtered according to size and aspect ratio ranges.

B. Initialization and Objects Distance Determination In this step, we initialize and define all the variables of Smart License Plate Character Detection algorithm include: license plate template with maximum 7 characters according to standard license plate format $\{\mathrm{C} 1, \mathrm{C} 2, \mathrm{C} 3$, $\mathrm{C} 4, \mathrm{C} 5, \mathrm{C} 6, \mathrm{C} 7\}, \mathrm{L}_{\mathrm{A}}$ as First character candidate $\left(\mathrm{L}_{\mathrm{A}} \epsilon\right.$ $\{C 1\})$ and $L_{B}$ as one of the rest of character candidate $\left(L_{B} \epsilon\right.$ $\{\mathrm{C} 2, \mathrm{C} 3, \mathrm{C} 4, \mathrm{C} 5, \mathrm{C} 6, \mathrm{C} 7\})$ except C1, Width and Length of $\mathrm{L}_{\mathrm{A}}$ and $\mathrm{L}_{\mathrm{B}}$ objects, Count, 'HDis' as Horizontal distance between center points of characters $\mathrm{L}_{\mathrm{A}}$ and $\mathrm{L}_{\mathrm{B}}$ and 'VDis' as Vertical distance between center points of characters $\mathrm{L}_{\mathrm{A}}$ and $\mathrm{L}_{\mathrm{B}}$ according to figure 1 and $\Theta$ define as angle between horizontal line and line between two center points of characters $L_{A}$ and $L_{B}$ that shows in figure 2 and calculate according to equation (1):

$$
\Theta=\tan ^{-1}\left(\frac{\text { VDis }}{\text { HDis }}\right)
$$

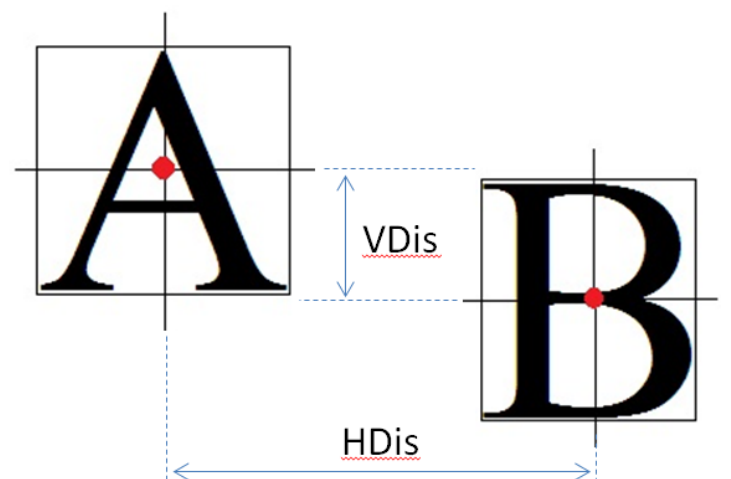

Fig 1.Horizontal (HDis) and Vertical (VDis) distance between two characters

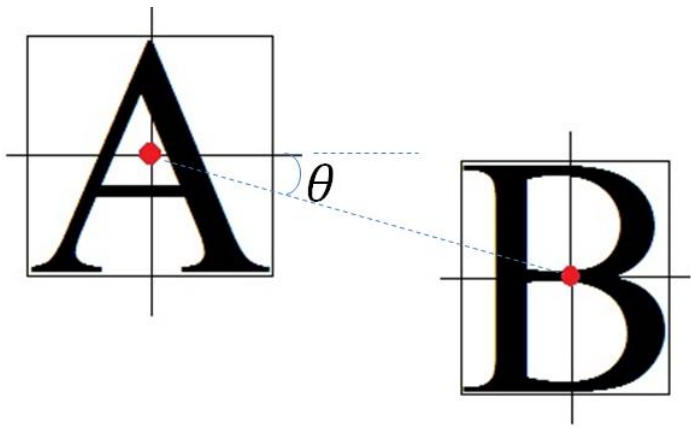

Fig 2.Angle between two characters

In license plate template each element of $\{\mathrm{C} 1, \mathrm{C} 2, \mathrm{C} 3, \mathrm{C} 4$, $\mathrm{C} 5, \mathrm{C} 6, \mathrm{C} 7\}$ includes character label from CCL stage to find and track the location of characters in input image and 
character segmentation data that will be used in Optical Character Recognition. 'Count' will determine the number of characters in each license plate; standard license plate has 7 characters ( 3 Letters and 4 numbers). To reach stable values for Vertical and Horizontal character distance, center of characters will be selected as reference because if other pixels for example minimum column pixel location of character (firs point of character) selected to calculate Horizontal distance then there could be some degradation on different character edges that affects Horizontal distance between two characters. In this research distance between license plate characters $\mathrm{L}_{\mathrm{A}}$ and $\mathrm{L}_{\mathrm{B}}$ on license plate standard template will be determined based upon on percentage amount of 'ChALength' as first character candidate's length (Length value of $\mathrm{L}_{\mathrm{A}}$ ), as an important advantage, this distance will be independent of license plate characters pixel size in an image. That's vital because the size of license plate characters is unknown and varies in different input images.

\section{Consecutive Objects and Character Counting}

To determine consecutive objects as license plate characters we create two searching loop on the CCL output objects $\left\{\mathrm{O} 1, \mathrm{O} 2, \ldots, \mathrm{O}_{\mathrm{n}}\right\}$, first loop searches for the first character candidate $\mathrm{C} 1$ as $\mathrm{L}_{\mathrm{A}}$ that $\mathrm{L}_{\mathrm{A}} \in\{\mathrm{O} 1, \mathrm{O} 2$, $\left.\ldots, \mathrm{O}_{\mathrm{n}-6}\right\}$ and second loop inside the first loop searches for $\mathrm{L}_{\mathrm{B}}$ as one of license plate characters except $\mathrm{C} 1$ include $\{\mathrm{C} 2, \mathrm{C} 3, \mathrm{C} 4, \mathrm{C} 5, \mathrm{C} 6, \mathrm{C} 7\}$ that $\mathrm{L}_{\mathrm{B}} \in\left\{\mathrm{O} 2, \mathrm{O} 3, \ldots, \mathrm{O}_{\mathrm{n}}\right\}$. This algorithm has 3 precondition steps:

Precondition Step 1, Width pixel value of $L_{A}$ and $L_{B}$ compared and the difference between widths of $\mathrm{L}_{A}$ and $\mathrm{L}_{\mathrm{B}}$ should be in the predefined range, based on experimental results on different alphabet and degraded images that license plate characters rotation angle $\Theta$ is $0<\theta<7$ degree, Width of characters will be changed according to equation (2):

$(0.7 *$ ChBWidth $<$ ChAWidth $<1.3 *$ ChBWidth $)$ or $(0.7 *$ ChAWidth $<$ ChBWidth $<1.3 *$ ChAWidth $)$

Where 'ChBWidth' is width of character $L_{B}$ and 'ChAWidth' is width of character $\mathrm{L}_{\mathrm{A}}$, according to experimental results this width difference range covers degraded images and rotated characters below 7 degree, but if we have rotated characters by rotation angle between $\alpha \in[7,20]$ in the input image then character's width variation range will be changed because when character rotates horizontal Width pixel of character will be changed accordingly based onFigure 3 .

To determine the relationship between L'length and $W^{\prime}$ width of rotated characters(character rotation angle $\alpha \in[7,20])$ and $\mathrm{L}$ length and $\mathrm{W}$ width values of nonrotated characters (character rotation angle $\alpha \in[0,7]$ ), we use definitions of sines and cosines to find lengths of $\mathrm{S}, \mathrm{T}$, $\mathrm{U}$ and $\mathrm{V}$ segments illustrated on Figure 3. In equation (3) assuming thatcharacter rotation angle $\alpha \in[7,20], \mathrm{L}>0$ and $\mathrm{W}>0$ :

$\operatorname{Cos} \alpha=\mathrm{S} / \mathrm{L}, \mathrm{S}=\mathrm{L} * \operatorname{Cos} \alpha, \operatorname{Sin} \alpha=\mathrm{T} / \mathrm{L}, \mathrm{T}=\mathrm{L} * \operatorname{Sin} \alpha$

$\operatorname{Cos} \alpha=\mathrm{U} / \mathrm{W}, \mathrm{U}=\mathrm{W} * \operatorname{Cos} \alpha, \operatorname{Sin} \alpha=\mathrm{V} / \mathrm{W}, \mathrm{V}=\mathrm{W} * \operatorname{Sin} \alpha$

Finally, relationship between rotated and non-rotated Length and Width of characters calculated in equation (4):

$$
\begin{aligned}
\mathrm{L}^{\prime} & =\mathrm{V}+\mathrm{S}=(\mathrm{W} * \operatorname{Sin} \alpha)+\left(\mathrm{L}^{*} \operatorname{Cos} \alpha\right) \\
\mathrm{W}^{\prime}=\mathrm{T}+\mathrm{U} & =\left(\mathrm{L}^{*} \operatorname{Sin} \alpha\right)+\left(\mathrm{W}^{*} \operatorname{Cos} \alpha\right)
\end{aligned}
$$

Therefore based on equation (4) Width range will be modified, minimum range is the same as equation (2) but maximum width change is calculated according to equation (4), where $\mathrm{L}=2.8 * \mathrm{~W}$ then $\mathrm{W}^{\prime}=(2.8 * \sin 20+\cos$ $20)^{*} \mathrm{~W} \cong 1.9 \mathrm{~W}$, final range of Width change for nonrotated and rotated characters $0<\theta<20$ shows in equation (5).

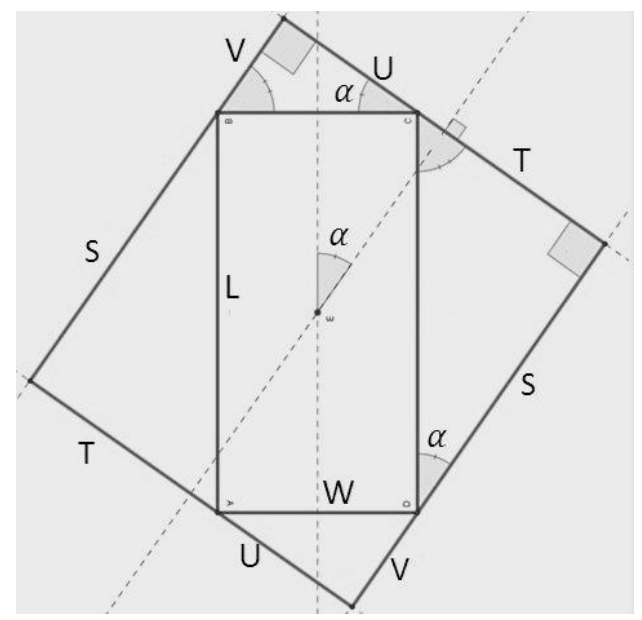

Fig 3. Non-rotated and rotated character dimensions

\section{$(0.7 *$ ChBWidth $<$ ChAWidth $<1.9 *$ ChBWidth $)$ or (0.7*ChAWidth $<$ ChBWidth $<1.9 *$ ChAWidth)}

Precondition Step 2, Length pixel value of $L_{A}$ and $L_{B}$ compared and the difference between lengths of $\mathrm{L}_{A}$ and $\mathrm{L}_{B}$ should be in the predefined range similar to precondition Step 1.For not-rotated license plate characters and rotation angle $\Theta$ between $0<\theta<7$ degree, length of characters will be changed according to equation (6):

$(0.8 *$ ChBLength $<$ ChALength $<1.2 *$ ChBLength) or $(0.2 *$ ChALength $<$ ChBLength $<1.2 *$ ChALength)

Where 'ChBLength' is length of character $L_{B}$ and 'ChALength' is length of character $\mathrm{L}_{\mathrm{A}}$. Based on equation (4.4) small amount of change happened for rotated length changed for character rotation angle $\alpha \in$ $[7,20]$ that slightly decreased the maximum range so equation (6) will be used for non-rotated and rotated characters $0<\theta<20$.

Precondition Step 3, according to standard license plate template, maximum distance between first character (C1) and last character (C7) is equal to ( $4 *$ ChALength), also maximum vertical distance between license plate characters 'MVDis' for rotation angle $0<\Theta<20$ degree calculated in equation (7):

$$
\begin{gathered}
\text { MVD is }= \\
\text { Tangent } 20^{\circ}(4 * \text { ChALength })=1.456 * \text { ChALength }(7)
\end{gathered}
$$

So whenever horizontal and vertical distance between $\mathrm{L}_{\mathrm{A}}$ and $\mathrm{L}_{\mathrm{B}}$ at the same time were in the range: (HDis> $(4 *$ ChALength $))$ and ((Vdis $>1.456 *$ ChALength) $)$ then $\mathrm{L}_{\mathrm{B}}$ object is far from first character candidate $\mathrm{L}_{\mathrm{A}}$ to include in license plate characters then we break the second loop 
International Journal of Advanced Research in Computer and Communication Engineering

Vol. 4, Issue 12, December 2015

and $\mathrm{L}_{\mathrm{A}}$ jump to the next object in first loop and process reset to search for license plate character from precondition Step 1.

When all three sequential precondition steps passed, horizontal and vertical distance between $\mathrm{L}_{\mathrm{A}}$ and $\mathrm{L}_{\mathrm{B}}$ as 'HDis' and 'VDis' and $\Theta$ angle between $L_{A}$ and $L_{B}$ will be calculate. Based on standard license plate size the vertical distance range between $\mathrm{L}_{\mathrm{A}}$ and $\mathrm{L}_{\mathrm{B}}$ defined as $[0$, tan $(\Theta)^{*}$ ChALength].

$\mathrm{L}_{\mathrm{A}}$ assumed as $\{\mathrm{C} 1\}$ first license plate character and based on horizontal and vertical distance between $\mathrm{L}_{\mathrm{A}}$ and $\mathrm{L}_{\mathrm{B}}$ we could determine $\mathrm{L}_{\mathrm{B}}$ as next license plate character from $\{\mathrm{C} 2, \mathrm{C} 3, \mathrm{C} 4, \mathrm{C} 5, \mathrm{C} 6, \mathrm{C} 7\}$. For $0<\Theta<7$ regarding to degraded images we assumed common 'VDis' range for all characters as $\left[0, \tan (7)^{*}\right.$ ChALength], if 'VDis' value was in predefined range then according to 'HDis' value, $\mathrm{L}_{\mathrm{B}}$ will be determined as $\mathrm{L}_{\mathrm{B}} \in\{\mathrm{C} 2, \mathrm{C} 3, \mathrm{C} 4, \mathrm{C} 5, \mathrm{C} 6, \mathrm{C} 7\}$. If 'HDis' was between [0.5*ChALength, $0.6^{*}$ ChALength] then $L_{B}$ will be assigned to second character $\{C 2\}$, otherwise if 'HDis' was in the range between [0.8*ChALength, $0.16^{*}$ ChALength] then $\mathrm{L}_{\mathrm{B}}$ will be assigned to character $\{\mathrm{C} 3\}$, otherwise if 'HDis' was in the range between [1.7*ChALength, $2.2 *$ ChALength] then $\mathrm{L}_{\mathrm{B}}$ will be assigned to character $\{\mathrm{C} 4\}$, otherwise if 'HDis' was in the range between $[2.3 *$ ChALength, $2.7 *$ ChALength] then $\mathrm{L}_{\mathrm{B}}$ will be assigned to character $\{\mathrm{C} 5\}$, otherwise if 'HDis' was in the range between [2.8*ChALength, $3.1 *$ ChALength] then $\mathrm{L}_{\mathrm{B}}$ will be assigned to character $\{\mathrm{C} 6\}$ and finally if 'HDis' was between range [3.2*ChALength, 3.8*ChALength] then $\mathrm{L}_{\mathrm{B}}$ will be assigned to last character $\{C 7\}$ then all the license plate characters already determined but if none of the distance range applied the next object in the second loop as $\mathrm{L}_{\mathrm{B}}$ will be selected and algorithm started from precondition Step 1 and repeat all the steps to fill out all the license plate characters $\{\mathrm{C} 1, \mathrm{C} 2, \mathrm{C} 3, \mathrm{C} 4, \mathrm{C} 5, \mathrm{C} 6, \mathrm{C} 7\}$. It is important to mention that whenever we reached $\{\mathrm{C} 7\}$ already all the license plate characters are determined and put all detected characters as Plate $1=\{\mathrm{C} 1 \mathrm{C} 2 \mathrm{C} 3 \mathrm{C} 4 \mathrm{C} 5$ $\mathrm{C} 6 \mathrm{C} 7\}$, then delete these characters $\{\mathrm{C} 1 \ldots \mathrm{C} 7\}$ from CCL object source and break from second loop and searching for Plate2 and whenever reached Plate2 algorithm is finished. For $7<\theta<20$ we will check the angle $\Theta$ that should be in the range of $5 \%$ variance for all characters candidates and Horizontal and Vertical distance will be checked based on the same predefined ranges.

According to simulation results on Matlab 2014A, table 1 illustrates processing time of 'Smart License Plate Character Detection' algorithm, on the 10 sample images based on this table we reached minimum computation time depends on complexity of image and number of objects in CCL algorithm. In [16] table 2 list different ALPR system based on complexity, processing time and image size. By comparing results of different ALPR methods with proposed method based on 'Smart License Plate Character Detection' algorithm we see that processing time improved significantly also proposed method work on different illumination condition based on proposed multiScale Adaptive NICK thresholding method.
TABLE I PRoCESSING TIME OF SMART LICENSE PlATE CHARACTER DETECTION

\begin{tabular}{|l|l|l|l|l|}
\hline $\begin{array}{l}\text { Im } \\
\#\end{array}$ & $\begin{array}{l}\text { Total } \\
\text { Objects }\end{array}$ & $\begin{array}{l}\text { Image } \\
\text { Resolution }\end{array}$ & $\begin{array}{l}\text { License } \\
\text { Plate }\end{array}$ & Time \\
\hline 1 & 311 & $1333 \times 1000$ & DFM6174 & 0.0033 \\
\hline 2 & 271 & $1333 \times 1000$ & DBY8082 & 0.0023 \\
\hline 3 & 311 & $1333 \times 1000$ & DDC5532 & 0.0045 \\
\hline 4 & 296 & $1333 \times 1000$ & CMV6593 & 0.003 \\
\hline 5 & 376 & $1333 \times 1000$ & DFM6174 & 0.0045 \\
\hline 6 & 916 & $1333 \times 1000$ & CMV6593 & 0.0077 \\
\hline 7 & 547 & $1333 \times 1000$ & DER2826 & 0.0044 \\
\hline 8 & 902 & $1333 \times 1000$ & CJY2021 & 0.0078 \\
\hline 9 & 458 & $1333 \times 1000$ & DFM6174 & 0.0045 \\
\hline 10 & 340 & $1333 \times 1000$ & CMV6593 & 0.0035 \\
\hline
\end{tabular}

TABLE II PERFORMANCE COMPARISON OF SOME TYPICAL ALPR SYSTEMS [LICENSE PLATE EXTRACTION (LPE), LiCEnSE Plate SEgMENTATION (LPS), OPTICAL CHARACTER RECOGNITION (OCR)] ACCORDING TO REFERENCE [16]

\begin{tabular}{|c|c|c|c|c|}
\hline $\begin{array}{l}\text { Ref } \\
\# \text { on } \\
{[16]}\end{array}$ & LPE & $\begin{array}{l}\text { Image } \\
\text { Conditions }\end{array}$ & $\begin{array}{l}\text { Processing } \\
\text { Time }\end{array}$ & $\begin{array}{l}\text { Plate } \\
\text { format }\end{array}$ \\
\hline [20] & $\begin{array}{l}\text { Hough } \\
\text { transform } \\
\text { and contour } \\
\text { algorithm }\end{array}$ & $\begin{array}{l}800 \times 600 \\
\text { pixels, } \\
\text { different } \\
\text { rotation and } \\
\text { lighting } \\
\text { conditions }\end{array}$ & $\begin{array}{l}0.65 \mathrm{~s} \text { for } \\
\mathrm{LPE} \text { and } \\
0.1 \mathrm{~s} \text { for } \\
\text { OCR }\end{array}$ & $\begin{array}{l}\text { Vietnamese } \\
\text { plates }\end{array}$ \\
\hline [18] & $\begin{array}{l}\text { Block-based } \\
\text { processing }\end{array}$ & $\begin{array}{l}\text { Multi plates } \\
\text { with } \\
\text { occlusion } \\
\text { and } \\
\text { different } \\
\text { sizes } \\
\end{array}$ & $\begin{array}{l}75 \mathrm{~ms} \text { for } \\
\text { LPE }\end{array}$ & $\begin{array}{l}\text { Taiwanese } \\
\text { plates }\end{array}$ \\
\hline [22] & $\begin{array}{l}\text { Edge } \\
\text { detection } \\
\text { and vertical } \\
\text { and } \\
\text { horizontal } \\
\text { projections }\end{array}$ & $\begin{array}{l}320 \times 240 \\
\text { pixels }\end{array}$ & $100 \mathrm{~ms}$ & $\begin{array}{l}\text { Taiwanese } \\
\text { plates }\end{array}$ \\
\hline [21] & $\begin{array}{l}\text { Edge } \\
\text { statistics } \\
\text { and } \\
\text { morphology }\end{array}$ & $\begin{array}{l}768 \times 534 \\
\text { pixels, } \\
\text { different } \\
\text { lighting } \\
\text { conditions }\end{array}$ & $100 \mathrm{~ms}$ & $\begin{array}{l}\text { Chinese } \\
\text { plates }\end{array}$ \\
\hline [49] & VQ & $\begin{array}{l}768 \times 256 \\
\text { pixels, } \\
\text { different } \\
\text { brightness } \\
\text { and sensor }\end{array}$ & $200 \mathrm{~ms}$ & $\begin{array}{l}\text { Italian } \\
\text { plates }\end{array}$ \\
\hline$[50]$ & SCW & $\begin{array}{l}\text { Different } \\
\text { background } \\
\text { and } \\
\text { illumination }\end{array}$ & $\begin{array}{l}276 \mathrm{~ms} \\
(111 \mathrm{~ms} \\
\text { for LPE, } \\
37 \mathrm{~ms} \text { for } \\
\mathrm{LPS}, \text { and } \\
128 \mathrm{~ms} \text { for } \\
\text { OCR) }\end{array}$ & $\begin{array}{l}\text { Greek } \\
\text { plates }\end{array}$ \\
\hline [62] & $\begin{array}{l}\text { Color and } \\
\text { fuzzy } \\
\text { aggregation }\end{array}$ & $\begin{array}{l}\text { Various } \\
\text { scene and } \\
\text { conditions }\end{array}$ & $\begin{array}{l}0.4 \mathrm{~s} \text { for } \\
\mathrm{LPE} \text { and } 2 \\
\mathrm{~s} \text { for OCR }\end{array}$ & $\begin{array}{l}\text { Taiwanese } \\
\text { plates }\end{array}$ \\
\hline
\end{tabular}

Finally, all the license plate characters for each license plate in image will be segmented to be used as an input for the 'Optical Character Recognition' System [15]. 
International Journal of Advanced Research in Computer and Communication Engineering

Vol. 4, Issue 12, December 2015

\section{CONCLUSION}

This paper presents improved Automated License Plate Recognition (ALPR) system based on 'Smart License Plate Character Detection' algorithm. Proposed Improved ALPR system is capable of distinguishing license plates under various conditions, such as distance from the camera, rotation angle between camera and vehicle (+/- 20 degree) and poor illumination condition (different weather condition, different lighting condition and physical tilted or damage of license plate). In our method, improved ALPR system has three main steps: 1) Image Enhancement, 2) Character Segmentation (Smart License Plate Character Detection Algorithm) and 3) Character Recognition. While in regular ALPR system there are four main steps: 1) Image Enhancement, 2) License Plate Location Extraction 3) Character Segmentation, 4) Character Recognition.

In thresholding and Binarization stage we used MultiScale Adaptive NICK thresholding method [1] to achieve all objects as characters candidates in the binary image, accuracy of this method is very important because extraction of license plate characters from input image depends on accuracy of image enhancement and thresholding method. As most advantage of Multi-scale Adaptive NICK thresholding method, it could be adapted for both types of degradation; Interfering Degradation and Intensity Degradation depends on scale or window size. At each scale, only binaries foreground pixels that are connected to the foregrounds pixels of the previous scales are labelled as character otherwise, they will be rejected.

After Image enhancement and thresholding by applying Single Pass Connected Components Labelling (CCL), put the label on detected objects then new design algorithm called "Smart License Plate Character Detection" finds the license plate characters directly in the input labelled binary image and eliminate the License Plate Location Extraction as complex and time consuming individual stage. Smart License Plate Character Detection algorithm detects the license plate characters regardless of license plate location, shape and background colour. Finally by utilize Optical Character Recognition [15] method license plate character will be recognize and save as text file in the database for further application. All the steps simulated in Matlab 2014A software and results of Smart License Plate Character Detection Algorithm illustrated on Table 1.

\section{REFERENCES}

[1] Rohollah Mazrae Khoshki, SubramaniamGanesan, "Multi-Scale Adaptive NICK Thresholding Method for ALPR system", International Journal of Advanced Research in Computer and Communication Engineering Vol. 4, Issue 10, October 2015

[2] Shan Du, Mahmoud Ibrahim, Mohamed Shehata and WaelBadawy," Automatic License Plate Recognition (ALPR): A State-of-the-Art Review", IEEE TRANSACTIONS ON CIRCUITS AND SYSTEMS FOR VIDEO TECHNOLOGY, VOL. 23, NO. 2, FEBRUARY 2013

[3] Mehmet Sezgin, BülentSankur, Mehmet Sezginb IMAGE THRESGOLDING TECHNIQUES: A SURVEY OVER CATEGORIES', Journal of Electronic Imaging 13(1), 146-165 (January 2004)

[4] NOBUYUKI OTSU, "A Tlreshold Selection Method from GrayLevel Histograms", IEEE TRANSACTIONS ON SYSTREMS,
MAN, AND CYBERNETICS, VOL. SMC-9, NO. 1, JANUARY 1979

[5] Niblack, W.: An Introduction to Digital Image Processing. Englewood Cliffs, N. J., Prentice Hall (1986) 115-116

[6] Sauvola, J., Pietikainen, M.: "Adaptive Document Image Binarization", Pattern recognition 33(2000), 225-236

[7] Reza FarrahiMoghaddam, Mohamed Cheriet, "A multi-scale framework for adaptive Binarization of degraded document images", Pattern Recognition 43 (2010) 2186-2198.

[8] KhurramKhurshid; Imran Siddiqi; Claudie Faure; Nicole Vincent, "Comparison of Niblack inspired Binarization methods for ancient documents",SPIE 7247, Document Recognition and Retrieval XVI, $72470 U$ (January 19, 2009)

[9] Soumen Bag, ParthaBhowmick, "Adaptive-interpolative Binarization with stroke preservation for restoration of faint characters in degraded documents", J. Vis. Commun. Image R. 31 (2015) 266-281

[10] Lifeng He, Yuyan Chao, Kenji Suzuki, "An efficient first-scan method for label-equivalence-based labeling algorithms", Pattern Recognition Letters 31 (2010) 28-35

[11] Ni Ma, Donald G. Bailey, Christopher T. Johnston, 'Optimized Single Pass Connected Components Analysis', 978-1-4244-27963/08/\$25.00 2008 IEEE

[12] Derek Bradley, Gerhard Roth, "Adaptive Thresholding Using the Integral Image", Journal of Graphics, GPU, and Game Tools Volume 12, Issue 2, 2007

[13] Xiehua Zhang, Xuzhou, "A robust license plate detection algorithm based on multi-features", Computer and Automation Engineering (ICCAE), 2010 The 2nd International Conference on (Volume:5 ), P 598 - 602, Feb. 2010

[14] DanianZheng, Yannan Zhao, Jiaxin Wang, "An efficient method of license plate location", Pattern Recognition Letters 26 (2005) 24312438, 22 June 2005

[15] William B. Lund, Eric K. Ringger "Improving Optical Character Recognition through Efficient Multiple System Alignment", JCDL '09 Proceedings of the 9th ACM/IEEE-CS joint conference on Digital libraries, Pages 231-240, NY, USA @2009

[16] Automatic License Plate Recognition (ALPR): A State-of-the-Art Review, 'IEEE TRANSACTIONS ON CIRCUITS AND SYSTEMS FOR VIDEO TECHNOLOGY, VOL. 23, NO. 2, FEBRUARY 2013'

\section{BIOGRAPHIES}

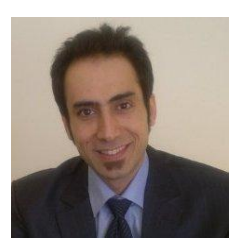

Rohollah Mazrae Khoshki, PhD student in Oakland University, Rochester, MI, USA, in Embedded System Electrical Eng. He investigated and designed of Interferometric Fiber Optic Gyroscope (IFOG) as his Master Thesis. He is interested in Embedded Systems, FPGAs and Microcontrollers, Robotics and control, Image processing and Optoelectronic in research and practical applications.

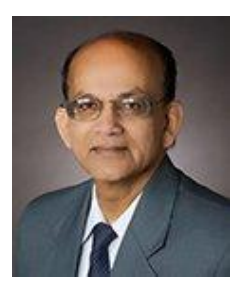

Dr. Subramaniam Ganesan is a Professor of Electrical and Computer Engineering, Oakland University, Rochester, MI, 48309, USA. He has over 25 years of teaching and research experience in Digital systems. He served as the chair of the CSE department from 1991 to 98 . He is with Electrical and Computer Engineering Department since 2008. He received his masters and Ph.D. from Indian Institute of Science (IISc) Bangalore, India. He worked at National Aeronautical Laboratory (NAL) India, Ruhr University, Germany, Concordia University Canada, and Western Michigan University before joining Oakland University in 1986 